\title{
The Role of Sucrose in the Development of Oral Biofilm in a Simulated Mouth System
}

\author{
Wan Nordini Hasnor Wan Ismail, Fathilah Abdul Razak and Zubaidah Hj Abdul Rahim \\ Department of Oral Biology, Faculty of Dentistry, University of Malaya \\ 50603 KUALA Lumpur, Malaysia
}

\begin{abstract}
The development of oral biofilm consisting of early settlers and late settlers in the presence and absence of sucrose using a simulated mouth model was investigated. The experiments were carried out by growing the early settlers' biofilm consisting of Strep. mitis and Strep. sanguinis and late settlers' biofilm of Strep. mutans in the simulated mouth system. Experiments using three different nutrient conditions (A, B and C) were carried out. In A, sterile saliva was used as the nutrient source in which the early settlers were suspended and pumped into the simulated mouth system and allowed to grow for $24 \mathrm{hrs}$. In B, $1 \%$ of BHI broth was added to the sterile saliva three times a day at $6 \mathrm{hrs}$ interval to demonstrate the effect of glucose on the development of the biofilms. In C, the $1 \%$ BHI was supplemented with $1 \%$ sucrose in order to show the effect of glucose in the presence of sucrose on the development of the biofilm. The experiments were subsequently repeated with the late settlers. In all the experiments, A serves as the control where the nutrient source is minimal. The data collected were analyzed using Student t-test. From the results obtained, it was observed that both glucose and sucrose enhanced the development of early and late settlers' biofilms. The growth of late settlers' biofilm however, was found to be significantly higher in the presence of sucrose $\left(93 \times 10^{6} \mathrm{CFU} \mathrm{mL} \mathrm{m}^{-1}\right)$ compared to glucose $\left(66 \times 10^{6} \mathrm{CFU} \mathrm{mL} \mathrm{m}^{-1}\right)$ with a $p$ value $<0.05$.
\end{abstract}

Key words: Early settlers' biofilm, late settlers' biofilm, simulated mouth system

\section{INTRODUCTION}

Sucrose yields one equivalent of glucose and one equivalent of fructose upon hydrolysis. In industrialized societies, sucrose is commonly used as sweetening agent and it had been reported that consumption of sucrose is about $50 \mathrm{~kg}$ per person per year ${ }^{[1]}$. Sucrose has been considered to be associated with dental caries. Sucrose can be utilized by extracellular bacterial enzymes for the formation of extracellular polysaccharides (glucans and fructans) in dental plaque $^{[1-4]}$. The formation of glucan is catalyzed by glucosyltransferase (GTF) and fructan by fructosyltransferase $(\mathrm{FTF})^{[5-7]}$. Glucan plays a role in plaque formation as well as facilitates the bacterial attachment to the tooth surface ${ }^{[8-10]}$. Fructan contributes to the virulence of the biofilm by acting as binding sites for the adhesion of Strep. mutans ${ }^{[7]}$. Sucrose consumption can result in a decrease in $\mathrm{pH}$ in both nonstarved and starved biofilms of Strep. mutans ${ }^{[11]}$.

The early development of oral biofilm (dental plaque) exhibits colonies of Streptococcus sanguinis and Streptococcus mitis ${ }^{[5,12]}$. Both streptococci belong to mitis-group ${ }^{[1,13]}$. Strep. sanguinis is capable of producing water-insoluble mutans and water-soluble dextran from sucrose $^{[1]}$ whereas Strep. mitis may produce water-soluble dextran ${ }^{[14]}$. In the oral cavity, these microbes usually exist as biofilm on the tooth surfaces and surrounded by protective matrix made up of extracellular polymers ${ }^{[1]}$. Strep. mutans on the other hand, belongs to mutans-group and has been associated with dental caries ${ }^{[13]}$. Dietary sucrose is essential for the accumulation of this group on teeth and initiation of carious lesions on smooth enamel surfaces ${ }^{[15]}$. Strep. mutans synthesized extracellular polysaccharide from sucrose by the action of two enzymes, GTF and FTF. When the bacteria are grown in the presence of any carbohydrate, addition of sterile sucrose to the culture results in the formation of extracellular polymers ${ }^{[16]}$. Strep. mutans produces three types of GTF that convert sucrose into glucans; (i) GTF synthesize water-soluble glucan, (ii) GTF synthesize water-insoluble glucan and (iii) GTF synthesize both types of glucan ${ }^{[10,17]}$.

The close relationship between sucrose, Strep. mutans and caries development had been well documented for centuries ${ }^{[2,18-21]}$. However, little or no information has been discussed on the role of carbohydrate in the formation of early settlers' biofilm. In this study, the role of glucose and sucrose on the growth of the oral biofilm consisting of early settlers (Strep. mitis and Strep. sanguinis) was investigated using a simulated mouth model and compared with that of the late settlers.

Corresponding Author: $\quad$ Prof. Dr. Zubaidah $\mathrm{Hj}$ Abdul Rahim, Department of Oral Biology, Faculty of Dentistry, University of Malaya, 50603 Kuala Lumpur, Malaysia, Tel/Fax: 603-79674851/603-79674536 


\section{MATERIALS AND METHODS}

Preparation of a simulated mouth model: The glass Pasteur-pipette was cut to $6 \mathrm{~cm}$ length using a diamond blade and was used as a chamber to mimic the oral cavity. Glass beads were placed in the chamber to serve as substratum for biofilm formation. The glass chamber was then connected to a peristaltic pump (Bio-Rad Econo Pump) with rubber tubing. The chamber was then immersed in a water bath, which functions as an incubator, with a temperature constantly monitored at $37^{\circ} \mathrm{C}$ to mimic the in vivo temperature of the mouth. The flow rate of all liquids through the artificial mouth

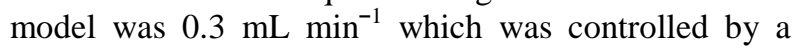
Bio-Rad Econo peristaltic pump.

Preparation of sterile saliva: Undiluted sterile saliva was prepared in a manner similar to that described previously by De Jong and Van Der Hoeven ${ }^{[22]}$. Approximately $25 \mathrm{~mL}$ of stimulated whole saliva (WS) was collected everyday from a single volunteer. The volunteer was asked to chew on a sugar-free gum to stimulate saliva production. The secreted stimulated WS was then collected using ice-chilled test tubes. The aggregation of protein in the saliva samples was reduced by adding 1, 4-Dithio-D, L-threitol (Fluka, BioChemika, Switzerland) (DTT) to a concentration of $2.5 \mathrm{mM}$. The saliva was then stirred slowly for $10 \mathrm{~min}$ and centrifuged (864 x g, $30 \mathrm{~min}$ ). The supernatant was filter-sterilized through a disposable $0.2 \mu \mathrm{m}$ (Supor ${ }^{\circledR}$ Membrane) low protein-binding filter (Acrodisc $®$ Syringe Filters, Pall Corp, USA) into sterile test tubes to give sterile saliva which was then stored at $-20^{\circ} \mathrm{C}$. Prior to use, the saliva was thawed and centrifuged once again to remove any precipitate.

Preparation of bacterial suspension: The stock of oral bacteria (Strep. mitis, Strep. sanguinis and Strep. mutans) from frozen stock was thawed at room temperature and prepared in a manner similar to that described previously by Palmer et al. ${ }^{[23]}$. $100 \mu \mathrm{l}$ of the stock was inoculated into $20 \mathrm{~mL}$ of sterilized Brain Heart Infusion (BHI) broth separately and incubated at $37^{\circ} \mathrm{C}$ for $18-24 \mathrm{hrs} .0 .3 \mathrm{~mL}$ of the starter cultures was transferred into $8 \mathrm{~mL}$ of fresh BHI broth and allowed to grow for $5 \mathrm{hrs}$ (Strep. sanguinis), $4 \mathrm{hrs}$ (Strep. mutans) and $3 \mathrm{hrs}$ (Strep. mitis) at $37^{\circ} \mathrm{C}$ as static cultures in an aerobic incubator to reestablish the exponential growth. The cultures were then centrifuged and the pellets were washed three times in phosphate-buffer saline (PBS) before adding into sterile saliva. The suspension of each bacterium of early settlers in the sterile saliva was adjusted to 0.072 absorbance at $550 \mathrm{~nm}$ (equivalent to $0.5 \times 10^{8}$ cells $\mathrm{mL}^{-1}$ ) using a spectrophotometer and mixed together prior to the experiment. For Strep. mutans, the suspension was adjusted equivalent to a population of $1.0 \times 10^{8}$ cells $\mathrm{mL}^{-1}$ (spectrophotometrically to 0.144 absorbance at $550 \mathrm{~nm}$ ).
The development of biofilms: The Strep. sanguinis and Strep. mitis mixture suspended in sterile saliva (A) in a ratio of $1: 1(60 \mathrm{~mL})$ was inoculated into the simulated mouth system at a rate of $0.3 \mathrm{~mL} \mathrm{~min}^{-1}$. A peristaltic pump was used to pump the inoculums into the simulated mouth system. The biofilm was allowed to develop on the glass beads for $24 \mathrm{hrs}$. This serves as a control in which the biofilm was grown in the presence of minimum nutrients. Similar procedure was repeated for Strep. mutans.

The development of biofilm in the presence of exogenous nutrients supplement: The above method was repeated with a periodic inoculation of BHI broth (1\%) which is the source of glucose to the developing biofilm (B). The method was then repeated with a periodic inoculation of BHI broth $(1 \%)$ supplemented with $1 \%$ sucrose (C). This was carried out to demonstrate the effect of glucose in the presence of sucrose on the development of the biofilm. The inoculation of nutrients was carried out three times a day at $6 \mathrm{hrs}$ interval for $24 \mathrm{hrs}$.

Assessment of the microbial load in biofilm: After 24 hrs, the glass beads with the biofilm were placed in a micro-centrifuge tubes containing $1.0 \mathrm{~mL}$ phosphatebuffered saline (PBS). The tubes were sonicated for a few seconds and vortexed for one minute to dislodge the bacteria from the surface of the glass beads. The microbial load was determined based on turbidity by reading the absorbance at 550nm using a spectrophotometer (UV-spectrophotometer 160A, SHIMADZU).

\section{RESULTS AND DISCUSSION}

Figure 1 shows the development of early settlers' biofilm consisting of Strep. mitis and Strep. sanguinis under three different nutrient conditions: A - Sterile saliva without the exogenous carbohydrate (Control), B - $1 \%$ broth containing glucose and C-1\% broth containing glucose and $1 \%$ sucrose.

In the absence of glucose or sucrose, minimum growth of early settlers' biofilm was observed. In the presence of glucose, the growth of the early settlers' biofilm was significantly enhanced by 4 -fold $(\mathrm{p}=0.03)$ and only a slight and insignificant increase (5\%) was observed in the presence of the added sucrose.

Figure 2 shows the development of late settlers' biofilm consisting of Strep. mutans under the three different nutrient conditions. In the absence of glucose and sucrose, the growth of late settlers' biofilm was found to be minimum compared to the growth of early settlers' biofilm. However, the difference is not significant. The growth of late settlers' biofilm was observed to be significantly enhanced by 8 -fold in the presence of glucose $(\mathrm{p}=0.00)$. 


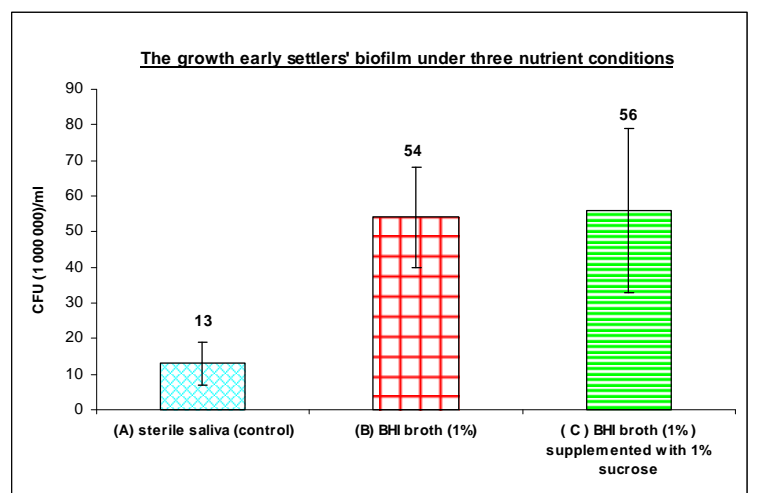

Fig. 1:The development of early settlers' biofilm (Strep. mitis and Strep. sanguinis) on glass beads placed in the simulated mouth model. The biofilm was allowed to develop on the glass beads for $24 \mathrm{~h}$. The values were expressed as means \pm SD. The number of determination $(\mathrm{n}=6)$. Experiment A-sterile saliva without exogenous carbohydrate (Control), Experiment B-sterile saliva with $1 \%$ broth containing glucose and Experiment $\mathrm{C}$-sterile saliva with 1 $\%$ broth containing glucose and $1 \%$ sucrose

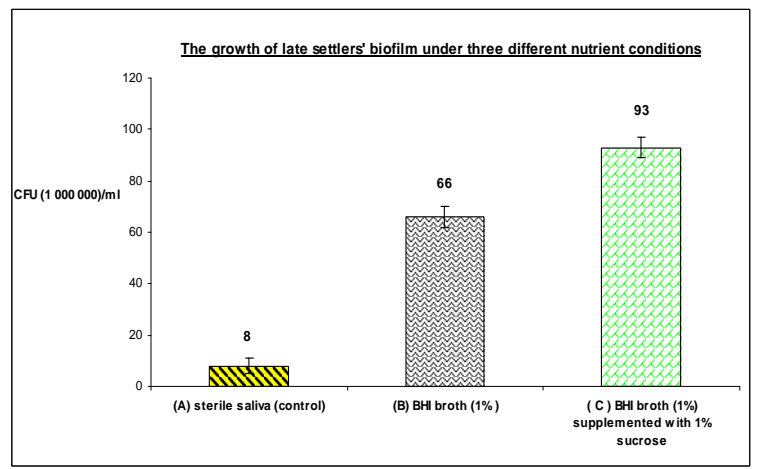

Fig. 2: The development of biofilm consisting of late settlers (Strep. mutans), on glass beads placed in the simulated mouth system. The biofilm was allowed to develop on the glass beads for $24 \mathrm{~h}$. The values were expressed as means \pm SD. The number of determination $(n=6)$. Condition Asterile saliva without exogenous carbohydrate (Control), Condition B-sterile saliva with $1 \%$ broth containing glucose and Condition C-sterile saliva with $1 \%$ broth containing glucose and 1 $\%$ sucrose

The presence of sucrose as in condition $\mathrm{C}$ was found to enhance the growth of the late settlers' biofilm significantly by 12 -fold $(\mathrm{p}=0.00)$.

From the Fig. 1 and 2, it was shown that glucose increased the growth of early and late settlers' biofilms to almost a similar degree (50-60 CFU x $10^{6} \mathrm{~mL}^{-1}$ ). Sucrose further enhanced the growth of late settlers' biofilm but not the early settlers' biofilm.

The purpose of the study was to look at the influence of nutrients supplement, specifically sucrose on the growth of both early settlers' and late settlers' biofilms. The sterile saliva used in the study was obtained from a single volunteer to minimize the variations that may arise. Although biofilms can be grown on various type of substratum ${ }^{[24-28]}$, glass beads were chosen because of its transparency features which make it easier to use in the system, as well as facilitate evaluation of samples. In addition, it has been reported by Pratt-Terpstra et al. ${ }^{[29]}$ that glass surfaces and enamel behave similarly with regard to oral microbial adhesion properties. It has also been shown that no difference was observed in the biofilm growth pattern when plain glass or hydroxyapatite coated glass was used as substratum $^{[30]}$. A periodic addition of exogenous carbohydrates to the sterile saliva was designed to mimic the intake of sugar in the diet.

Minimum development of the early settlers' biofilm was observed in the absence of exogenous carbohydrate. Under such condition, the saliva was used as the sole source of nutrient. This is in accordance with the findings of Theilade and Theilade $^{[31]}$ who showed that diet plays a minor role in the initiation of plaque formation. Their study showed that the nutrient requirement during the initial stage of plaque formation is sufficiently provided by the saliva. It has also been reported that oral streptococci can grow continuously in human whole saliva ${ }^{[22]}$ at the expense of the various organic components present in the saliva (particularly mucous glycoproteins) that support their growth $^{[32]}$. In addition to that, several species of oral streptococci have the ability to bind to salivary amylase which may help them to degrade salivary glycoproteins for direct metabolism ${ }^{[22,33]}$.

Additional nutrients can influence the development of the oral biofilm. It was observed that the presence of glucose in the growth environment stimulated the growth of the early settlers' biofilm (Fig. 1). Bacteria in the developing biofilm may utilize the supplemented glucose to support their growth. This explains the 4fold increase observed in Fig. 1. This is in agreement with the observation by Shera et al. ${ }^{[34]}$ that the growth of Group A Streptococci biofilm is influenced by glucose. Therefore, the biofilm containing streptococci groups is utilising glucose for growth.

The results obtained in this study appear to indicate that sucrose is not a crucial factor for the development of biofilm consisting of early settlers as it only caused a slight increase (5\%). The slight increase in the biofilm observed in this study may agree with earlier observation that sucrose slightly stimulated the adhesion of Strep. sanguinis ${ }^{[35]}$ and can cause the in vitro formation of small amounts of plaque ${ }^{[36]}$.

A study carried out by Pratten et al. ${ }^{[37]}$ contradicts the result obtained in this study. This might be due to the source of the inoculums. Pratten et al. ${ }^{[37]}$ has used non sterile saliva as inoculums. The inoculums of non sterile saliva would provide a multi-species biofilm contrary to the inoculums of sterile saliva used in this study. 
The development of late settlers' biofilm under minimal nutrients (sterile saliva) was lower compared to that of the early settlers' biofilm. It has been shown that unlike the early settlers' biofilm, the development of the late settlers' biofilm is influenced by nutrients ${ }^{[38,39]}$. This may explain the lower growth of the late settlers observed in the absence of exogenous nutrients.

In the presence of glucose, the growth of late settlers was significantly increased compared to the control $(\mathrm{p}<0.05)$. Compared to glucose, the addition of sucrose further increased the growth $(\mathrm{p}<0.05)$. This agrees with what had been reported by Cury et al. ${ }^{[4]}$ that plaque wet weight was significantly higher in dental plaque formed in the presence of sucrose than in the presence of glucose and fructose. From this study, it is obvious that sucrose greatly enhanced the growth of Strep mutans. This is in accordance with what have been reported earlier ${ }^{[40-42]}$.

From this study, it can be concluded that sucrose has a role in the development of the late settlers' biofilm but not the early settlers' oral biofilm. The study also demonstrated the versatility of the simulated mouth system designed and indicated that oral biofilm has been successfully developed in the simulated mouth system.

\section{ACKNOWLEDGEMENT}

This study was supported by Vote F0145/2005A, R\&D IRPA No 09-02-03-0197 EA197 \& Kuok Foundation Bhd.

\section{REFERENCES}

1. Marsh, P. and Martin M.V., 1999. Oral Microbiology. $4^{\text {th }}$ Ed. Great Britain: Wright.

2. Rolla, G., Scheie A.A. and Ciard J.E., 1985. Role of sucrose in plaque formation. Scand. J. Dent. Res., 93: 105-111.

3. Schonfeld, S.E., 1992. Oral Microbial Ecology. In Slots, J. and Taubman, M.A. (Ed.), Contemporary Oral Microbiology and Immunology. St. Louis: Mosby Year Book, pp: 267-274.

4. Cury, J.A., Rebelo M.A.B., Del Bel Cury A.A., Derbyshire M.T.V.C. and Tabchoury C.P.M., 2000. Biochemical composition and cariogenicity of dental plaque formed in the presence of sucrose or glucose and fructose. Caries Res., 34: 491-497.

5. Maiden, M.F.J., Lai C-H. and Tanner A., 1992. Characteristics of Oral Gram-positive Bacteria. In Slots, J. and Taubman, M.A. (Ed), Contemporary Oral Microbiology and Immunology. St. Louis: Mosby Year Book, pp: 342-372.

6. Marsh, P. and Martin, M.V., 1992. Oral Microbiology. $3^{\text {rd }}$ Ed. London: Chapman \& Hall.
7. Rozen, R., Bachrach, G. and Steinberg, D., 2004. Effects of carbohydrates on fructosyltransferase expression and distribution in Streptococcus mutans GS-5 biofilms. Carbohydrate Res., 339: 2883-2888.

8. Schilling, K.M. and Bowen, W.H., 1988. The activity of glucosyltransferase adsorbed onto saliva-coated hydroxyapatite. J. Dent. Res., 67: 28.

9. Schilling, K.M. and Bowen, W.H., 1992. Glucans synthesized in situ in experimental salivary pellicle function as specific binding sites for Streptococcus mutans. Infect. Immun., 60: 284-295.

10. Ooshima, T., Matsumura, M., Hoshino, T., Kawabata, S., Sobue, S. and Fujiwara, T., 2001. Contributions of three glucosyltransferases to sucrose-dependent adherence of Streptococcus mutans. J. Dent. Res., 80: 1672-1677.

11. Zhu, M., Takenaka, S., Sato, M. and Hoshino, E., 2001. Influence of starvation and biofilm formation on acid resistance of Streptococcus mutans. Oral Microbiol. Immunol., 16: 24-27.

12. Fathilah, A.R. and Rahim, Z.H.A, 2003. The antiadherence effect of Piper betle and Psidium guajava extracts on the adhesion of early settlers in dental plaque to saliva-coated glass surfaces. J. Oral Sci., 45: 201-206.

13. Samaranayake, L.P., 2002. Essential Microbiology for Dentistry $2^{\text {nd }}$ ed. Edinburgh: Churchill Livingstone.

14. Melville, T.H. and Russell, C., 1975. Microbiology for Dental Students $2^{\text {nd }}$ ed. London: William Heinemann Medical Books Ltd.

15. Bleiweis, A.S. and Oyston, P.C.F., 1993. Adhesion and Cohesion of Plaque Microflora: A Function of Microbial Fimbriae and Fibrils? In Bowen, W.H. and Tabak, L.A. (ed), Cariology for the Nineties. United Kingdom: University of Roschester Press, pp: 287-299.

16. Tanzer, J.M., 1992. Microbiology of Dental Caries. In Slots, J. and Taubman, M.A. (ed), Contemporary Oral Microbiology and Immunology. St. Louis: Mosby Year Book, pp: 377-424.

17. Banas, J.A. and Vickerman, M.M., 2003. Glucanbinding proteins of the oral streptococci. Crit. Rev. Oral Biol. Med., 14: 89-99.

18. Staat, R.H., Gawronski, T.H., Cressey, D.E., Harris, R.S. and Folke, I.E.A., 1975. Effects of dietary sucrose levels on the quantity and microbial composition of human dental plaque. J. Dent. Res., 54: 872-880.

19. Killian, M. and Rolla G., 1976. Initial colonization of teeth in monkeys as related to diet. Infect. Immun., 14: 1022-1027.

20. Van Houte, J., 1994. Role of microorganisms in caries etiology. J. Dent. Res., 73: 672-681. 
21. Cury, J.A., Marques A.S., Tabchoury C.P.M. and Del Bel Cury A.A., 2003. Composition of dental plaque formed in the presence of sucrose and after its interruption. Braz. Dent. J., 14: 147-152.

22. De Jong, M.H. and Van Der Hoeven J.S., 1987. Growth of oral bacteria on saliva. J. Dent. Res., 66: 498-505.

23. Palmer, J.R.Jr., Kazmerzak, K., Hansen, M.C. and Kolenbrander P.E., 2001. Mutualism vs Independence: strategies of mixed-species oral biofilms in vitro using saliva as the sole nutrient source. Infect. Immun., pp: 5794-5804.

24. Sudo, S.Z., 1977. Continuous culture of mixed oral flora on hydroxyapatite-coated glass beads. Appl. Environ. Microbiol., pp: 450-458.

25. Sissons, C.H., Cutress T.W., Hoffman M.P. and Wakefield J.St.J., 1991. A multi-station dental plaque microcosm (artificial mouth) for the study of plaque growth, metabolism, $\mathrm{pH}$ and mineralization. J. Dent. Res., 70: 1409-1416.

26. Sissons, C.H., Cutress T.W., Faulds G. and Wong L., 1992. $\mathrm{pH}$ responses to sucrose and the formation of $\mathrm{pH}$ gradients in thick 'artificial mouth' microcosm plaques. Arch. Oral Biol., 37: 913-922.

27. Herles, S., Olsen, S., Afflitto, J. and Gaffar, A., 1994. Chemostat flow cell system: an in vitro model for the evaluation of anti plaque agents. J. Dent. Res., 73: 1748-1755.

28. Pratten, J., Smith, A.W. and Wilson, M., 1998. Response of single-species biofilms and microcosm dental plaques to pulsing with chlorhexidine. J. Antimicrob. Chemother., 42: 453459.

29. Pratt-Terpstra, I.H., Weerkamp, A.H. and Busscher H.J., 1989. The effects of pellicle formation on streptococcal adhesion to human enamel and artificial substrata with various surface freeenergies. J. Dent. Res., 68: 463-467.

30. Elliott, D., Pratten, J., Edwards, M., Crowther, J., Petrie, A. and Wilson, M., 2005. Bacterial biofilm development on hydroxyapatite-coated glass. Curr. Microbiol., 51: 41-45.

31. Theilade, E. and Theilade, J., 1985. Formation and ecology of plaque at different locations in the mouth. Scand. J. Dent. Res., 93: 90-95.
32. Bowen, W.H., 1996. Salivary Influences on the Oral Microflora. In Edgar, W.M. and O'Mullane, D.M. (Ed.), Saliva and Oral Health London: British Dental Association, pp: 95-103.

33. Douglas, C.W.I., 1994. Bacterial-protein interactions in the oral cavity. Adv. Dent. Res., 8: 254-262.

34. Shera, J., Sriprakash, K.S. and McMillan, D.J., 2006. The nutritional requirements for biofilm formation by Group A streptococcus. Intl. Congr. Ser., 1289: 139-142.

35. Adshead, V.M., Parke, J.M., Chambers, P.J., Davies, R.M. and Cole, J.A., 1983. An in vitro study of the role of sucrose and interactions between oral bacteria in possible mechanisms of dental plaque formation. Arch. Oral. Biol., 28: 723727.

36. Socransky, S.S., Manganiello, A.D., Propas, D., Oram, V. and Van Houte, J., 1977. Bacteriological studies of developing supragingival dental plaque. J. Periodontal. Res., 12: 90-106.

37. Pratten, J., Andrews, C.S., Craig, D.Q.M. and Wilson, M., 2000. Structural studies of microcosm dental plaques grown under different nutritional conditions. FEMS Microbiol. Lett., 189: 215-218.

38. Wennerholm, K., Birkhed, D. and Emilson, C.G., 1995. Effects of sugar restriction on Streptococcus mutans and Streptococcus sobrinus in saliva and dental plaque. Caries Res., 29: 54-61.

39. Renye, J.A.Jr., Piggot, P.J., Daneo-Moore, L. and Buttaro, B.A., 2004. Persistence of Streptococcus mutans in stationary-phase batch cultures and biofilms. Appl. Environ. Microbiol., 70: 61816187.

40. Seppä, L., Pöllänen, L. and Hausen, H., 1988. Streptococcus mutans counts obtained by a dipslide method in relation to caries frequency, sucrose intake and flow rate of saliva. Caries Res., 22: 226-229.

41. Karjalainen, S., Karjalainen, M. and Söderling, E., 1993. Effect of sucrose rinses on the oral microflora and on salivary sucrase activity. Caries Res., 27: 38-42.

42. Cury, J.A., Francisco, S.B., Del Bel Cury, A.A. and Tabchoury, C.P.M., 2001. In situ study of sucrose exposure, mutans streptococci in dental plaque and dental caries. Braz. Dent. J., 12: 101-104. 